\title{
Thoracic Oncologic Surgery in Covid-19 Pandemic: Short Term Outcomes
}

\author{
Zeynep Bilgi \\ Medeniyet University, Faculty of Medicine, Department of Thoracic Surgery, Istanbul, Turkey. \\ Correspondence Author: Zeynep Bilgi \\ E-mail: zeynep.bilgi@gmail.com \\ Received: $13.06 .2021 \quad$ Accepted: 16.09 .2021
}

\begin{abstract}
Objective: After sustained community spread of Coronavirus Disease - 19 (COVID-19), guidelines regarding safe practice are still evolving. In this article, we evaluated our clinic's oncologic thoracic surgery practice in COVID-19 pandemic via an observational retrospective study.

Methods: All cases (including local anesthesia only), were recorded in the hospital electronic medical record system. Patients undergoing procedures for cancer related causes (resection, diagnosis, palliation, treatment facilitation) were retrospectively identified. Patient demographic data, type of operation/procedure, 30 day SARS-COV-2 PCR test data, hospital readmission due to COVID-19, 30 and 90 day mortality were gathered. Group 1 and 2 were operated between 1 June 2019 - 11 March 2020 and 12 March 2020 - 31 December 2020, respectively.

Results: 344 (Group 1= 178, Group 2=166) patients underwent thoracic surgery due to cancer related causes during the study period. Surgical or COVID-19 related mortality were not encountered in either group. After initiation of mandatory testing, 3 asymptomatic patients were found to be PCR positive and had surgery rescheduled (2 lung resections, 1 mediastinoscopy). Number of lung resection cases increased postCOVID-19 (25 vs 45, p<0.001). None of the postoperative patients experienced PCR conversion.

Conclusion: Safe elective thoracic surgery practice is possible via following basic safety precautions and following evolving national guidelines. Even though routine preoperative SARS-COV-2 PCR testing catches asymptomatic cases that can cause mortality/morbidity for patients and staff, social distancing and other infection control measures have to be in place to prevent in hospital and post-operative SARS-COV-2 PCR conversion.
\end{abstract}

Keywords: COVID-19 pandemic, Elective surgical procedure, Lung cancer, Thoracic surgery

\section{INTRODUCTION}

Since early 2020, COVID-19 pandemic has forced substantive change in surgical practices all over the world. Elective surgical procedures are restricted in many parts of the world due to the conservation of limited resources (manpower, personal protective equipment, ventilator, intensive care bed) and resource distribution for acute needs, maintaining patient and employee safety (1). In places where the epidemic can be controlled, elective procedures can continue with various security measures (2). However, the benefit/risk analysis of delaying surgery due to COVID-19 is still controversial for some particular surgical practices (cardiovascular surgery, oncologic surgery) or safe amount of wait time for certain patient groups which are more problematic in terms of urgency, when for example, compared to elective knee or hip replacement is not well defined (3).
The risk of clinically serious events was found to be higher in patients who were operated in the month before contracting SARS-CoV-2 compared to those who did not have surgery (4). Currently, there are no published international guidelines for decisions regarding specific considerations for surgical intervention in cancer patients during the pandemic (5). However, lung cancer was observed with a rate of $28 \%$ in patients with a diagnosis of malignancy among COVID-19 patients (4), so lung cancer patients stand out as a particularly vulnerable group in the pandemic population.

Although there are flow charts and suggestion booklets created by many experts on this subject, limited field knowledge on specific surgical branches delays creation of an evidence based guideline $(1,2,5)$. 
The objective of this study is to summarize and characterize our clinic's experience of thoracic oncologic surgery during COVID-19 pandemic.

\section{METHODS}

Patients who underwent thoracic surgery due to cancer related causes (resection, diagnosis, palliation, treatment facilitation) between June 12019 and December 312020 were identified from the hospital electronic medical record system and included in the study. $11^{\text {th }}$ March (identification of first COVID-19 case in Turkey) was chosen for separation of the pre (Group 1) and post (Group2) COVID-19 groups. Patient admissions, demographic parameters (age, sex, in or out of province), type of surgery, presence of SARS-COV-2 screening, length of hospital stay, postoperative complications, 30 and 90 day mortality, COVID related postoperative morbiditymortality during follow up were recorded.

SARS-COV-2 PCR was mandatory after $15^{\text {th }}$ July, so was performed for every case with planned general anesthesia. Otherwise it was left to surgeon discretion. Cases without planned general anesthesia were only directed to testing in case of clinical suspicion (contact history, symptoms, radiologic findings).

All patients underwent standard preoperative testing and clinical staging work-up, including pulmonary function tests. Informed consent was introduced for pulmonary function tests, per health ministry quality guidelines. Post pandemic, staging and preoperative testing were done in as few days possible to prevent exposure to public transport etc.

Data was analyzed using SPSS 22.0. $p<0.05$ was used for statistically significance. Mean, standard deviation, median values were calculated accordingly. Kolmogorov Simirnov test was used to determine distribution appropriate choice for comparison of independent quantitative variables.

Institutional ethical board (decision number 2020/0754) and Ministry of Health approval were obtained before conduction of the study.

\section{RESULTS}

344 patients underwent cancer related thoracic surgery during the study period. 178 patients (101 male, 77 female) were assigned to Group 1 (pre - COVID), 166 patients (107 male, 59 female) were assigned to Group 2 (post-COVID). Mean age was $55.1 \pm 14$ and $56.1 \pm 15.3$ for groups 1 and 2 respectively. Sex and age distribution were comparable among groups ( $p>0.05$ for both, Chi-Square and MannWhitney-U respectively). 41 patients in Group 1, 37 patients in Group 2 traveled to Istanbul from out of province to get treatment ( $p>0.05$, Chi-Square).

Preoperative evaluation via American Society of Anesthesiologists (ASA) Physical Status Classification System revealed that both groups were equivalent. Significantly more patients underwent lung resection (anatomic and nonanatomic) in Group 2 ( 25 vs 45, p=0.002, Chi-Square), while chest wall resections and mediastinal procedures were found to be decreased ( 13 vs $1, p=0.001$ and 22 vs 9, $p=0.02$, ChiSquare). Number of chemotherapy port insertions, pleural diagnostic/palliative procedures, lymph node biopsies were comparable among groups. Operation type breakdown is provided in Table 1.

Table 1: Patient characteristics and operation type breakdown and mortality

\begin{tabular}{|c|c|c|c|}
\hline & Group 1 & Group 2 & $\mathrm{p}$ Value \\
\hline Age & $55.1 \pm 14.0$ & $56.1 \pm 15.3$ & 0.30 \\
\hline Sex (Male/Female) & $101 / 77$ & $107 / 59$ & 0.17 \\
\hline ASA class $(1 / 2 / 3 / 4)$ & $31 / 125 / 21 / 1$ & $8 / 130 / 28 / 0$ & $\mathrm{~N} / \mathrm{A}$ \\
\hline Operation type & $\begin{array}{l}\text { Chemotherapy port }=96 \\
\text { Lung Resection }=25^{*} \\
\quad \text { Lung resection with mediastinal lymph node dissection } \\
=15 \\
\quad \text { Wedge }=10 \\
\text { Mediastinal }=22 \\
\quad \text { Mediastinoscopy }=12 \\
\quad \text { Resection }=10 \\
\text { Lymph node biopsy }=16 \\
\text { Pleural = } 6 \\
\text { Chest wall resection }=13^{*}\end{array}$ & $\begin{array}{l}\text { Chemotherapy port }=93 \\
\text { Lung Resection }=45^{*} \\
\quad \text { Lung resection with mediastinal lymph node dissection } \\
=25 \\
\quad \text { Wedge }=10 \\
\text { Mediastinal }=9 \\
\quad \text { Mediastinoscopy }=6 \\
\text { Resection }=3 \\
\text { Lymph node biopsy }=10 \\
\text { Pleural }=8 \\
\text { Chest wall resection }=1 *\end{array}$ & $* p<0.05$ \\
\hline Mortality (n) & $\begin{array}{l}19 \\
30 \text { day mortality=5 } \\
90 \text { day mortality=4 }\end{array}$ & $\begin{array}{l}4 \\
30 \text { day mortality=0 } \\
90 \text { day mortality=1 }\end{array}$ & $P=0.002$ \\
\hline
\end{tabular}


In both groups, chemotherapy port cases and lymph node biopsies were conducted as day case operations, 2 and 5 chemotherapy port cases in Group 1 and 2 respectively and 6 lymph node biopsies in both groups needed inpatient stay for symptom control/palliation. Lung resection cases had a comparable length of stay $(8.3 \pm 5.6$ vs $7.7 \pm 5.9$ days, $p>0.05$, Mann-Whitney-U).

43 cases (20 under general anesthesia, 10 lung resections) were operated after the identification of the first COVID-19 patient in the country but before the health ministry guideline change regarding mandatory SARS-COV-2 PCR screening. 1 patient (anatomic lung resection) underwent SARS-COV-2 PCR testing due to post-operative clinical and radiologic deterioration at that period and found to be negative, recuperated well afterwards. No post-operative COVID-19 related mortality and morbidity was observed.

Rest of the Group 2 was operated under current policy, with a negative SARS-COV-2 PCR test within 48 hours of the operation, 3 asymptomatic and radiology negative patients were found to be positive and had their surgery postponed for 2-3 weeks (2 lung resections, 1 mediastinoscopy) and readmitted after a negative PCR for the rescheduled procedure, none of those patients experienced further adverse events.

Significantly more deaths were recorded in Group 1 (19 vs 4, $\mathrm{p}=0.002$, Chi-Square), 14 of 19 deaths in Group 1 occurred in chemotherapy port cases and were not attributable to the procedure itself. There was no mortality observed in either group's lung resection cases.

\section{DISCUSSION}

After declaration of COVID-19 as a global health emergency in January 2020, first case was identified in Turkey at $11^{\text {th }}$ March 2020. Since the beginning of the pandemic, COVID-19 has put a considerable strain on health services as a whole, affecting staffing levels, inpatient bed and ICU capacity, need for personal protective equipment etc. Many countries, including Turkey, introduced unprecedented measures when compared to the last 7 decades, to limit the contagion so the health systems are not overwhelmed and the resources are appropriately prioritized.

Careful evaluation and determination of the severity of population level spread is very important for setting appropriate guidelines for preoperative screening and case selection. In a recent case series from Aminian et al, illustrates the point with 3 cases of subclinical COVID-19 patients undergoing elective surgeries for cholecystectomy, hernia repair and hysterectomy. Two operations were conducted right before the identification of the index case in the country, one right after. 1 patient died post-operatively, also another fatality happened for a scheduled gastric bypass surgery on the planned day, as the patient arrived to emergency with respiratory distress (6).

Since surgical needs of oncology patients are time sensitive and has less tolerance for deferral, also considering the waxing/waning nature of COVID-19 together with precautionary measures, many expert groups tried to come up with prioritization and screening protocols as exampled by Center for Disease Control (CDC), ASA and Anesthesia Patient Safety foundation (APSF) (7). These institutions also advise for universal symptom and contact checking for all cases and standard preoperative PCR testing if the are prevalence is high.

In terms of classification of surgery types for appropriate prioritization, Stahel reported 5 main groups as with deferral times as urgent ( $<24$ hours), urgent elective ( $<2$ weeks), Essential elective (1-3 months), Discretionary elective (indefinite). Cardiothoracic operations and Cancer surgery/ biopsies were classified as urgent elective and essential elective respectively $(9,10)$.

3 asymptomatic, PCR positive cases were identified after introduction of mandatory testing and were operated on uneventfully afterwards. While the decision to postpone surgery and re-test for clearance of infection seems straightforward for both patient and staff safety, exact timelines were not apparent at the time of the decision and we moved forward per infectious disease clinic consultations. Further literature upon the subject do advice up to 7 weeks of interval after SARS-COV-2 infection in the absence of risk of disease progression (11). To consider an oncology predominant cohort, Baiocchi et al did a case control study where 49 cases with a positive PCR test for SARS-COV-2 (9 symptomatic) underwent surgery with a median of 25 days from the positive PCR test, with a comparable complication profile with the control cases, but their cohort is mostly formed by general surgery cases (12).

In-hospital precautions are also as important as pre-admission testing of SARS-COV-2. In a report summary encompassing over 70000 patients, Wu et al (13) emphasize that even though it is not responsible for most of the cases, one of the leading settings for secondary infections is hospitals and health workers are the ones mostly at risk in this scenario.

Cases with either cancer or emergency related causes are predominantly represented in normal thoracic surgery practice as there are very few other type operations (thoracic symphatectomy, chest wall deformity correction etc) that are within the scope of thoracic surgery. So it may be considered as expected to have near normal surgical volume during this pandemic. We did experience decreased 30 and 90 day mortality due to decrease in mortality after chemotherapy port insertions as well, since particularly morbid/advanged stage patients might have postponed this comfort measure due to COVID-19.

Due to dynamic and flexible organization and resource prioritization with multidisciplinary and administrative collaboration, thoracic oncologic procedures of radical intent, diagnosis, palliation and treatment facilitation were able to be performed without changing the rhythm of the normal workflow, as evidenced by largely comparable case volume and composition. Health ministry preoperative screening 
guidelines were adequate in preventing COVID-19 related postoperative morbidity/mortality without needing extra precautions. Also mobility restrictions enforced throughout the country did not significantly affect the number of out of province patients accessing our services. This experience might not be universal as Wang et al describe decreased oncologic case volume decreases and a backlog of at least 6 weeks worth of cases after a peak time of COVID-19 (January-April 2020), and underlines the need of adaptation of precautionary measures as pandemic evolves (eg: keeping the preoperative PCR test but dropping thorax computerized tomography and blood count requirement after the infection curve is flattened) for more straightforward access and costeffectiveness (14).

This study is conducted in a single center setting, therefore is limited in case numbers and events captured, including an assessment of overall surgical mortality and morbidity. Further preferably prospective studies are needed to delineate the effects of COVID-19 on thoracic surgery practice and patient population to ensure timely diagnosis and treatment.

\section{CONCLUSION}

Thoracic oncologic practice can be safely continued during COVID-19 pandemic while abiding by simple precautions. Prospective studies are needed to answer important questions like how the health service accessibility can be improved. While complete preoperative testing is possible during COVID-19 pandemic, facilitation can be achieved with protected time slots for those vulnerable oncology patients. Centralized systems should offer guidance regarding COVID-19 prevalence in particular healthcare system's population to determine optimal pre-operative PCR testing strategy.

Funding: No funding was used for conduction of this study

Conflicts of interest: The author does not have any conflict of interest regarding the material presented in the study.

Data Sharing: The author will make anonymized data available per request.

\section{REFERENCES}

[1] Diaz A, Sarac BA, Schoenbrunner AR, Janis JE, Pawlik TM. Elective surgery in the time of COVID-19. Am J Surg. 2020;219(6):900-902.

[2] Glasbey JC, Bhangu A, COVIDSurg Collaborative. Elective Cancer Surgery In COVID-19-Free Surgical Pathways During The SARS-Cov-2 Pandemic: An International, Multicenter,
Comparative Cohort Study. Journal Of Clinical Oncology, 2021;36(1):66-78.

[3] Shehata IM, Elhassan A, Jung JW, Urits I, Viswanath O, Kaye AD. Elective cardiac surgery during the COVID-19 pandemic: Proceed or postpone?. Best Pract Res Clin Anaesthesiol. 2020;34(3):643-650.

[4] Liang W, Guan W, Chen R et al. Cancer patients in SARSCoV-2 infection: A nationwide analysis in China. Lancet Oncol 2020;21:335-337.

[5] Al-Shamsi HO, Alhazzani W, Alhuraiji A, Coomes EA, Chemaly $\mathrm{RF}$, Almuhanna M. A Practical Approach to the Management of Cancer Patients During the Novel Coronavirus Disease 2019 (COVID-19) Pandemic: An International Collaborative Group. Oncologist. 2020;25(6):e936-e945.

[6] Aminian A, Safari S, Razeghian-Jahromi A, Ghorbani M, Delaney CP. COVID-19 Outbreak and Surgical Practice: Unexpected Fatality in Perioperative Period. Ann Surg. 2020;272(1):e27-e29.

[7] Centers for Disease Control and Prevention, National Center for Immunization and Respiratory Diseases (NCIRD), Division of Viral Diseases (2020) Discontinuation of transmissionbased precautions and disposition of patients with COVID-19 in healthcare settings (Interim Guidance). https://www.cdc. gov/coronavirus/2019-ncov/hcp/disposition-hospitalizedpatients.html Accessed 30 Dec 2020

[8] American Society of Anesthesiologists (ASA) and the Anesthesia Patient Safety foundation (APSF) (2020) ASA and APSF Joint Statement on Perioperative Testing for the COVID-19 Virus. https://www.asahq.org/about-asa/ newsroom/news-releases/2020/04/asa-and-apsf-jointstatement-on-perioperative-testing-for-the-covid-19-virus. Accessed 30 Dec 2020

[9] Stahel P.F. How to risk-stratify elective surgery during the COVID-19 pandemic? Patient Safety in Surgery. 2020:14-18.

[10] Al-Omar K, Bakkar S, Khasawneh L, Donatini G, Miccoli P. Resuming elective surgery in the time of COVID-19: a safe and comprehensive strategy. Updates Surg. 2020;72(2):291-295.

[11] El-Boghdadly K, Cook TM, Goodacre T, Kua J, Blake L, Denmark S. SARS-CoV-2 infection, COVID-19 and timing of elective surgery. Anaesthesia, 2021;76:940-946.

[12] Baiocchi, G, Aguiar, S, Duprat, JP, Coimbra FJF, Makdissi FB, Vartanian JG. Early postoperative outcomes among patients with delayed surgeries after preoperative positive test for SARS-CoV-2: A case-control study from a single institution. J Surg Oncol. 2021;123:823- 833. https://doi.org/10.1002/ jso. 26377

[13] Wu Z, McGoogan JM. Characteristics of and important lessons from the coronavirus disease 2019 (COVID-19) outbreak in China: Summary of a report of 72314 cases from the Chinese Center for Disease Control and Prevention. JAMA. 2020;323(13):1239-1242.

[14] Wang R, Zhong R, Liang H, Zhang T, Zhou X, Huo Z. Thoracic surgery and COVID-19: changes and managements during the pandemic. J Thorac Dis. 2021;13(3):1507-1516. 